\title{
Proteger la naturaleza en España y en Francia. Un análisis comparado del proceso de aplicación de la directiva europea Hábitat
}

\section{Sylvia Becerra}

LMTG-CNRS (Toulouse, Francia)

becerra@lmtg.obs-mip.fr

\section{Tomás García González}

Universitat Autònoma de Barcelona tgarcia@uab.cat

\section{Eduardo Moyano Estrada}

IESA-CSIC (Córdoba, España)

emoyano@iesa.csic.es

\section{Resumen}

En este artículo, sus autores analizan el proceso de aplicación de la Directiva europea Hábitat en espacios naturales incluidos en la red Natura 2000. Concretamente, estudian varios casos en Francia y en España, comparando el sistema político-administrativo y la dinámica social surgida en torno a la definición del espacio natural objeto de protección y a la puesta en marcha del programa europeo destinado a la conservación de la biodiversidad. En primer lugar, se compara el procedimiento utilizado en ambos países para la definición de los llamados «lugares de interés comunitario» (LIC), mostrando cómo en Francia se propicia la participación de los grupos sociales implicados, mientras que en España son los departamentos de medio ambiente de los gobiernos regionales los que asumen el principal protagonismo. En segundo lugar, se analiza en cada caso el grado de vertebración de los grupos sociales, comprobando hasta qué punto se produce o no una conciliación de intereses en torno al espacio natural protegido. La principal conclusión de la investigación realizada consiste en afirmar que no es el sistema administrativo ni la voluntad conservacionista de los departamentos de medio ambiente el factor que ejerce mayor influencia en el éxito de las políticas ambientales, sino el contexto socioeconómico local, de modo que allí donde se produce una conciliación previa de intereses en torno a un equilibrio entre conservación de la naturaleza y desarrollo económico, hay más posibilidades de que se pueda dar una adecuada gestión del programa.

Palabras clave: medio ambiente, políticas públicas, sociedad civil, acción colectiva.

Abstract. Conservation of Nature in Spain and France. Comparative Analysis of the implementation process of the European Directive Habitats

This article analyses the implementation process of the EU Directive Habitats in natural areas belonging to the network Nature 2000. Particularly, its authors study some cases in France and Spain, comparing the administrative systems and the social dynamics involved in the implementation of this European policy, which is designed to foster the conservation of biodiversity. Firstly, the procedures used in both countries to define the so-called LIC are 
compared; in this respect, it is shown that in France, the government has sought the participation of various social groups, whereas in Spain, the implementation process has been dominated by the environmental agencies of regional governments. Secondly, the interaction between interest groups is analysed in order to see if they manage to balance conflicting interests regarding the natural area concerned. The study's main conclusion is that in both countries, the social and economic context at the local level, rather than the administrative system or the conservationist attitudes of government agencies, constitutes the key for explaining the success or failure of environmental policies. Consequently, the probability of success in terms of program implementation is higher in areas where, a bargaining process among local interest groups has previously generated a suitable equilibrium between environmental protection and economic development interests.

Key words: environment, public policies, civil society, collective action.

\section{Sumario}

Introducción Els Aiguamolls del Empordà

Metodologia

La aplicación del programa Natura 2000 en Francia y en España

Análisis de los casos de estudio

Las lagunas de Bages-Sigean (Cataluña, España)

Las marismas de la bahía de Cádiz y del río Palmones (Andalucía, España)

Conclusiones

Referencias bibliográficas

(región Languedoc-Roussillon, Francia)

\section{Introducción}

En Europa, la conservación de los espacios naturales se ha convertido en un elemento fundamental de lo que se denomina la «ruralidad postmoderna» (Jollivet, 1997). En ese contexto, la pérdida de biodiversidad no sólo preocupa a la comunidad científica, sino que también se ha trasladado al ámbito de la opinión pública. Encuestas de reconocido prestigio, como el Ecobarómetro de Andalucía, vienen mostrando que la desaparición de especies vegetales y animales es uno de los problemas ambientales que más preocupa a la población ${ }^{1}$. Tal sensibilidad ante el problema de la pérdida de biodiversidad sintoniza con la convicción de que el desarrollo humano depende, desde el punto de vista social y económico, pero también cultural e identitario, de la existencia de una elevada variedad de especies vegetales y animales en los espacios naturales.

1. Desde el primer Ecobarómetro de Andalucía, realizado por el IESA-CSIC en 2000, el problema de la desaparición de especies vegetales y animales viene situándose de forma recurrente en un segundo bloque de problemas entre las preocupaciones ambientales de los andaluces (detrás de la capa de ozono, pero al mismo nivel que el cambio climático). Uno de cada tres andaluces sitúa este problema entre los dos más importantes en materia de medio ambiente (Jiménez y Moyano, 2005). 
Fruto de ello ha sido la creación, a nivel de la Unión Europea (UE), de la red Natura 2000, una trama de espacios naturales protegidos en los que, mediante la aplicación conjunta o separada de las directivas comunitarias Aves (Dir. 409/1979) y Hábitat (Dir. 43/1992), se pretende asegurar la supervivencia a largo plazo de las especies animales y vegetales más amenazadas en el territorio europeo, sintonizando así con los principios fijados en la Cumbre Mundial de Johannesburgo respecto al problema de la pérdida de biodiversidad. La red Natura 2000 está formada por espacios catalogados como «zonas de especial protección de aves» (zonas ZEPA; en francés ZPS) —en los que interviene la Directiva Aves— o bien como «zonas especiales de conservación» (zonas ZEC; en francés ZCS) —-sometidos a la Directiva Hábitat—. También existen áreas en las que ambas directivas actúan conjuntamente ${ }^{2}$.

La red Natura 2000 es hoy el principal instrumento de la UE para la conservación de la naturaleza en el territorio europeo, habiendo sido incluida como eje $2^{\circ}$ del desarrollo rural en el reglamento FEADER recientemente aprobado (Reg. 1698/2005). Con esta inclusión, la dimensión ambiental se integra en el conjunto de la política de desarrollo rural, en una especie de síntesis entre agricultura, naturaleza y territorio con la que la UE quiere impregnar sus orientaciones en esta materia (Moyano, 2005). Sin embargo, el hecho de que la inclusión de una zona en la red Natura 2000 suponga importantes restricciones al uso del correspondiente espacio natural con fines económicos (sean agrícolas, industriales o turísticos), explica la controversia surgida en torno a este asunto, ya sea en la fase de propuesta de los LIC, ya sea en la fase de gestión del territorio una vez decretada su calificación como ZEPA o ZEC.

Esto explica también que la preocupación general por el problema de la biodiversidad no sea compartida con la misma intensidad en todos los países, tal como lo indican las variaciones que se observan en la aplicación de las directivas europeas en los territorios de la red Natura 2000. En efecto, según un estudio realizado a principios del año 2004 por la Comisión Europea (CE, 2004), parece que, en general, la aplicación de la Directiva Hábitat no significa la adopción de medidas efectivas de conservación en los espacios seleccionados, y que se producen, además, retrasos significativos en su puesta en marcha ${ }^{3}$.

2. La integración de esos espacios naturales en la red Natura 2000 exige el reconocimiento previo de la Comisión Europea, que, a propuesta de los gobiernos de los estados miembros, elabora una lista de lugares de interés comunitario (LIC) para cada país. Una vez publicada esa lista, los gobiernos tienen que aprobar un decreto para designar un área determinada como espacio de la red Natura 2000, sometiéndolo a las normas de las directivas Aves o Hábitat (o ambas), según las características de la protección que se pretende en tales áreas.

3. Como prueba de ese retraso, baste comentar que, a final del año 2000, la mayor parte de los estados miembros de la UE no habían elevado sus propuestas de LIC a la Comisión Europea (cuando se había previsto que estuviera terminada esa fase en junio de 1995). Aún, en mayo de 2002, había países (como Alemania y Francia) cuyas propuestas de LIC eran calificadas por la Comisión Europea de "claramente insuficientes». 
Esta situación de cierta parálisis, o al menos de escaso dinamismo, en la puesta en marcha de las directivas Aves y Hábitat cobra sentido si consideramos que la dificultad de su aplicación refleja muchos de los problemas planteados por la intervención pública en el ámbito de las políticas de conservación de la naturaleza. Si bien es cierto que las directivas europeas son normas jurídicas de obligado cumplimiento para todos los estados miembros de la UE, también lo es que el ritmo y la modalidad de su aplicación se deja a la libre elección de los gobiernos nacionales. De ahí que su aplicación en el correspondiente territorio nacional exija, de una parte, la traducción previa al ordenamiento jurídico de cada país - lo que representa un primer punto de dificultad- $\mathrm{y}$, de otra, la existencia de una infraestructura técnica y administrativa a nivel nacional, adaptada a las exigencias normativas de cada directiva - lo que representa el segundo factor de dificultad (Izcara, 1999)—. Además, a nivel local, la aplicación de las políticas de conservación de la naturaleza se enfrenta, a veces de manera muy directa, a intereses económicos, así como a prácticas, usos, valores, tradiciones o inercias sociales, que se resisten a los cambios (Whitby, 1996). Las dinámicas de movilización en torno a la protección de la biodiversidad se convierten, por tanto, en un escenario complejo, a veces conflictivo, pero siempre sometido a lógicas de intereses donde los actores implicados calculan los costes y los beneficios antes de emprender cualquier tipo de movilización.

Asimismo, el carácter impreciso y difuso de los objetivos implícitos en las políticas de conservación de la naturaleza, así como el carácter muchas veces incierto de las consecuencias directas o indirectas de estas políticas en la explotación y valorización de los espacios naturales a los que van dirigidas, limitan el grado de adhesión que reciben por parte de los actores locales (Padioleau, 1993). Así, por ejemplo, el grupo de los que sufren el coste de la protección de una zona húmeda - como ocurre con los propietarios de explotaciones agrarias o los cazadores, por ejemplo, que ven restringidas sus prácticas de uso del espacio natural-, reacciona de forma diferente a como lo hace el beneficiario potencial de esas políticas de intervención — la población mundial, las generaciones futuras...- - Sea como fuere, el cálculo estratégico que realizan los actores implicados es efectuado a partir de un sistema de valores y de referencias simbólicas, específico para cada categoría de actores, lo que explica, en parte, que la movilización sea desigual según los grupos sociales involucrados. A ello habría que añadir el contexto político y mediático, cuya influencia en la diversidad de las movilizaciones no puede considerarse en absoluto despreciable (Mann, 1991).

La disimetría en las constricciones y en los beneficios ligados a la salvaguarda de la biodiversidad, constituye un elemento fundamental para explicar las diferencias en las dinámicas de acción colectiva que se desarrollan en este ámbito, lo que introduce un factor de incertidumbre en la eficacia esperada de las medidas concretas de conservación (Nevers, 2002). Tal disimetría estimula los componentes oportunistas e incita a las prácticas de free-rider (o gorroneo), tan bien estudiadas por la sociología a raíz del trabajo pionero de Mancur 
Olson (1965). En efecto, en este ámbito, la acción colectiva produce unos resultados (la conservación de la naturaleza, el mantenimiento de la biodiversidad...) que tienen carácter de "bien público», en el sentido de beneficiar a todos los individuos, tanto a los que participan directamente en las movilizaciones, como a los que no lo hacen ni asumen los costes de la cooperación.

\section{Metodologia}

La investigación empírica que sirve de base a este artículo se enmarca en la tesis doctoral desarrollada por uno de los autores (Becerra, 2003a) dentro del programa de doctorado de la Universidad francesa de Toulouse-Le Mirail. El trabajo de campo consistió en la realización de siete estudios de caso, en los que, con una finalidad exploratoria, se revisó en una primera fase una amplia documentación jurídica y administrativa, y se consultaron archivos de prensa referidos a las zonas seleccionadas. La información de carácter primario se recogió durante la estancia de varios meses (en los años 2000 y 2002) de la doctoranda en las zonas de estudio - bajo la orientación de los otros dos autores de este artículo-, bien observando in situ las dinámicas surgidas en torno al programa de conservación en cada área, bien realizando un programa de entrevistas semiestructuradas a distintas categorías de informantes (responsables políticos, técnicos y administrativos responsables de la ejecución de los programas, usuarios, representantes de los colectivos afectados, etc. $)^{4}$.

Cuatro de esos estudios se realizaron en Francia. Tres de ellos en la región Midi-Pyrennées, en sendas zonas húmedas del tramo del río Garona entre la ciudad de Toulouse y su confluencia con el río Tarn (la zona del lago de SaintNicolas de la Grave, el espacio natural de Monbequi y la zona húmeda de Gagnac), ocupando una superficie de 14.105 ha (todas ellas zonas ZEPA y ZEC); el cuarto estudio se realizó en la región Languedoc-Roussillon, en un área del litoral mediterráneo (el complejo lagunar de Bages-Sigean) de 9.555 ha (también reconocidas como zonas ZEPA y ZEC).

Los tres estudios restantes se efectuaron en España. Uno en Cataluña, en el parque natural de Els Aiguamolls de l'Empordà (declarado como zona ZEPA en sus 4.731 ha, pero de las que sólo 231 habían sido incluidas como zona ZEC); y dos en Andalucía, en el parque natural de la bahía de Cádiz (10.395 ha) y en el paraje natural del río Palmones, en la comarca gaditana del Campo de Gibraltar (57 ha), todas ellas reconocidas en su totalidad como zonas ZEPA y ZEC.

En cada una de las siete áreas seleccionadas se analizaron los diversos usos del espacio natural y las formas de vertebración social generadas en torno a

4. El trabajo de campo desarrollado por Sylvia Becerra en Cataluña se realizó en colaboración con Tomás García (Universidad Autónoma de Barcelona), y el realizado en las dos comarcas de Cádiz se llevó a cabo bajo la supervisión de Eduardo Moyano (IESA-CSIC). Una ampliación de las bases metodológicas de la investigación, así como la lista de las personas entrevistadas, pueden verse en la propia tesis doctoral (Becerra, 2003a). 
tales usos. Asimismo, se estudió el contexto económico y político en cada zona y el modo como se estaba aplicando el programa público de conservación, así como los resultados alcanzados a nivel local.

\section{La aplicación del programa Natura 2000 en Francia y en España}

La aplicación del programa Natura 2000 plantea un problema más general, a saber: cómo hacer compatible, por un lado, la protección de la naturaleza y, por otro, el mantenimiento de los usos, las tradiciones y las prácticas sociales que influyen en la puesta en valor de los recursos naturales, exigencia que, desde el artículo 8 del acuerdo firmado en la Cumbre de Río (1991), se considera un elemento indiscutible para la conservación de la biodiversidad.

En Francia, el Gobierno se adhirió rápidamente al objetivo prioritario de la UE de frenar, en el horizonte del año 2010, la pérdida de biodiversidad. En ese sentido, definió las orientaciones generales de una estrategia de carácter nacional que debía ser «implementada en concertación con todos los departamentos ministeriales (agricultura, turismo, ordenación del territorio, investigación...) y con todos los actores implicados, principalmente los concejales de los municipios afectados y demás cargos electos». Esta estrategia, definida en 2004, establecía el compromiso del Gobierno francés de crear un marco adecuado para la aplicación de las distintas políticas destinadas a la protección de los espacios naturales y la conservación de las especies vegetales y animales ${ }^{5}$, con lo que se inscribía en la lógica de "gestión integrada» que había caracterizado desde hacía veinte años a las políticas francesas de conservación de la naturaleza. En este tipo de intervenciones, se ha procurado tener en cuenta tanto las funciones naturales de los espacios a proteger, como los diferentes usos que les da la población, prestando especial atención a los procesos de concertación con los grupos afectados y favoreciendo la participación de la ciudadanía. Eso explica que, en la estrategia francesa por la biodiversidad, se haya incluido como objetivo prioritario la participación social en la toma de decisiones y se hayan tenido en cuenta las nuevas demandas de la población y las experiencias adquiridas en el modo de gestionar los conflictos surgidos en materia de medio ambiente.

En España, el marco de elaboración y aplicación de las políticas ambientales ha sido diferente, tanto en su planteamiento inicial como en el plano jurídico-institucional y político. Lo primero que llama la atención es que la primera medida de protección de la naturaleza aparece, de manera precoz, en 1916, con la aprobación de la ley de parques nacionales, que permanecerá en vigor hasta 1957. Lamentablemente, la aplicación de esta ley se hizo sin la participación de la ciudadanía, ya que, en el contexto de la dictadura franquista, la eje-

5. Nota de la rueda de prensa del ministro francés de Ecología y Desarrollo Sostenible (10 de septiembre de 2003). 
cución de esta política se canalizaba de forma exclusiva a través del personal funcionario del correspondiente ministerio, a lo que había que añadir la lógica debilidad de la sociedad civil española para autoorganizarse y la imposibilidad, por razones políticas, de construir su propio sistema de representación de intereses. De este modo, el gobierno de turno elaboraba las políticas públicas sin apenas contar con la sociedad civil (Aguilar-Fernández, 1997). La caída de la dictadura y la aprobación de la Constitución de 1978 conducirían a la reconfiguración del sistema político-administrativo y al abandono del modelo centralizado y arbitrario de acción pública vigente hasta entonces, lo cual ofreció cauces para la participación de la ciudadanía en la gestión de los asuntos públicos.

Desde el punto de vista de las políticas de medio ambiente, la transferencia de competencias desde la Administración central hasta las nuevas administraciones regionales (comunidades autónomas) (en adelante, CC.AA.) rompió la lógica centralizadora e intervencionista del franquismo, pero creó una situación marcada por lo que algunos autores han denominado «reparto conflictivo de poderes» entre ambos niveles administrativos del Estado (AguilarFernández, Font y Subirats, 1999), una distribución de competencias que se iba produciendo a distintos ritmos y conforme se construía el propio Estado de las Autonomías. En ese contexto, la política de medio ambiente se desarrolló de modo diferente de unas regiones a otras, lo cual añadió un elemento de lentitud y complejidad a todo el proceso. De hecho, algunos gobiernos de las CC.AA. crearon sus propios departamentos de Medio Ambiente cuando todavía a nivel estatal no se había creado el correspondiente ministerio. En este sentido, cabe señalar que España fue el último país de la UE en dotarse de una estructura administrativa específica en materia de medio ambiente para el conjunto del territorio nacional, hecho que tuvo lugar en 1996, cuando el gobierno de centro-derecha del Partido Popular, presidido por José Ma Aznar, creó el Ministerio de Medio Ambiente. A ello habría que añadir que, hasta mediados de los años ochenta, la política de medio ambiente mantenía viva la lógica conservacionista heredada del periodo anterior, una lógica basada en acciones correctivas de la naturaleza mediante dispositivos de carácter reglamentista.

Si se analiza la fase de elaboración de las listas de los LIC de la red Natura 2000 en España y Francia, puede apreciarse con detalle la influencia de sus estructuras político-administrativas respectivas, así como del distinto estilo de las políticas francesa y española en materia de medio ambiente, estilos marcados, como hemos señalado, por diferencias sensibles en lo que respecta a favorecer la participación social en la definición de las áreas incluidas en la red de espacios naturales del programa europeo. A nivel nacional, lo primero que destaca entre ambas políticas es la magnitud de la superficie afectada, siendo muy superior en el caso español. En efecto, España propuso una de las listas de LIC más amplias del programa: el $14,8 \%$ de su territorio (marítimo y terrestre) en la primera propuesta (1999), aumentada al 22,6\% en 2006. Por el contrario, Francia, además de congelar el programa durante un año, propuso una de las 
listas más reducidas: en la propuesta inicial (1999) incluyó un 4,9\% de su territorio, y se mantuvo en ese nivel restrictivo siete años más tarde (sólo aumentado al 6,9\% en el año 2006).

Para explicar esas diferencias, habría que recurrir a varios factores. Entre ellos, destacan de manera significativa el diferente nivel de vertebración de la sociedad rural afectada — muy bajo en España y muy elevado en Francia- y la distinta capacidad de movilización de sus equipos representativos —en este caso, agricultores, organizaciones agrarias y demás grupos de intereses presentes en los correspondientes espacios naturales- a la hora de reaccionar ante el programa (Garrido-Fernández, 2000; Garrido-Fernández y Moyano, 2004). En efecto, en España, el bajo nivel de vertebración del mundo rural y la consiguiente ausencia de debate público en las fases iniciales del programa Natura 2000, hicieron que la definición de las áreas LIC y la posterior aplicación de la Directiva Hábitat siguieran una lógica administrativista de base territorial (definida desde arriba por los gobiernos regionales), con lo que no se propició la participación de la población potencialmente afectada, como sí ocurrió en el caso francés. Cabe recordar que los gobiernos regionales de las diecisiete CC.AA. españolas tienen competencias plenas en la aplicación de la política ambiental europea, y que al Ministerio de Medio Ambiente sólo le corresponde desempeñar un papel de coordinación entre las regiones y de intermediación con la Comisión Europea. En lo que se refiere a la elaboración de la propuesta de LIC española, todos los gobiernos regionales han actuado de forma similar, a saber: definiendo, desde los correspondientes departamentos de medio ambiente o similares, su propia red de espacios naturales protegidos —en muchos casos, superpuestas a redes previas ya existentes_- sin propiciar un debate serio y participativo con los grupos potencialmente afectados. De haberse producido, ese debate hubiera hecho implicarse a estos grupos en el programa desde sus fases iniciales y habría permitido a los responsables de la Administración regional recabar información sobre el terreno para evitar la inclusión en la red Natura 2000 de áreas de difícil gestión.

$\mathrm{Al}$ no haberse hecho así, la lista de LIC se elaboró en el marco de un procedimiento muy administrativista y con la indiferencia (por desconocimiento o desinterés) de la población afectada. Ello explica la ya comentada amplitud de la lista española de espacios protegidos, en la cual se reflejaban las diversas voluntades políticas implicadas y la diversidad de recursos humanos y financieros destinados al programa por los gobiernos regionales. Además, el hecho de que las organizaciones agrarias españolas estuvieran entonces poco preocupadas por los temas agroambientales y más por los temas de la reforma del primer pilar de la PAC (Garrido-Fernández, 2000), explica que el programa Natura 2000 pasara prácticamente desapercibido por el mundo agrícola, a pesar de sus importantes consecuencias para la agricultura, y explica también que el procedimiento de selección de los lugares LIC se llevara a cabo sin apenas resistencia por parte del mundo agrícola y rural, y según las prioridades fijadas en cada región por el cuerpo de funcionarios de los departamentos de medio ambiente. 
La aplicación del programa Natura 2000 en Francia estuvo, por el contrario, marcado, desde sus fases iniciales, por una fuerte movilización de los distintos actores del mundo agrícola, tanto a nivel nacional, primero, como a nivel regional, más tarde, y a nivel local, en su etapa final. Esa movilización fue propiciada no sólo por la ya comentada tradición participativa de las políticas francesas de medio ambiente (Duran, 2001), sino también por el efecto rechazo que provocó la perspectiva demasiado científica utilizada en la fase inicial - a partir de inventarios realizados por los expertos del Museo de Historia Natural de París (Charles y Kalaora, 1999)_, una perspectiva que, además de ser difícilmente comprensible por la población, además de generar recelos entre los grupos afectados, daría lugar a tensiones entre los departamentos ministeriales implicados ${ }^{6}$. Durante el proceso de elaboración de la lista de LIC, los representantes de los intereses agrarios y forestales franceses, así como del mundo de la caza y la pesca, se movilizaron y articularon sus fuerzas, creando incluso una plataforma a nivel nacional denominada "grupo de los nueve» $y$ siendo reconocidos como interlocutores privilegiados ante los responsables de la Administración. Fruto de su presión, lograron que la competencia relativa a la aplicación del programa Natura 2000 fuera asumida por el propio primer ministro, con lo cual se sacó de la esfera de los departamentos de medio ambiente (Alphandery y Fortier, 1999).

De este modo, la puesta en marcha de la Directiva Hábitat en las áreas incluidas en la red Natura 2000 francesa fue resultado, a diferencia de lo ocurrido en España, de un complejo proceso de concertación previa entre el Gobierno y los representantes de la sociedad civil organizada. Esto explica que la lista de LIC propuesta por el Gobierno francés a la Comisión Europea estuviera constituida por un reducido número de áreas, si bien todas ellas estaban definidas de forma consensuada con la población local y caracterizadas por la búsqueda de un equilibrio entre la conservación del espacio natural y el desarrollo económico del territorio circundante. En el plano jurídico, esta lógica de consenso se plasmaría en la Orden del 11 de abril de 2001 (título III), que convertía en obligatoria la necesidad de concertar con la población las medidas de conservación de los LIC en Francia, y que modificaba incluso el Código Rural francés.

En resumen, las diferencias observadas entre los casos francés y español en la elaboración de sus respectivas listas nacionales de LIC son resultado, de una parte, del modo como cada gobierno ha valorado la participación de la sociedad civil en la puesta en marcha de una política pública — en este caso, la política ambiental ligada al programa Natura 2000 - y, de otra, de la permeabilidad de cada sistema administrativo nacional a la presión ejercida por los distintos grupos de intereses - especialmente, de los que manifestaban actitudes abier-

6. La perspectiva utilizada ponía de manifiesto que el estado ya no es el único responsable de la definición de las políticas relacionadas con la protección de la naturaleza, porque el territorio constituye el lugar donde se redefinen los problemas de conservación de la biodiversidad (Billaud, Alphandery, Fortier y Pinton, 2002). 
tamente hostiles al programa europeo-, consecuencia a su vez de la capacidad de influencia de estos grupos. Así, mientras que, en el caso francés, fue necesario conciliar el interés general de proteger la naturaleza con los intereses más sectoriales de los grupos locales afectados, en el caso español, por el contrario, la ausencia de una sociedad civil vertebrada a nivel de cada territorio hizo que prevaleciera el interés general tal como era entendido por los departamentos de medio ambiente de los gobiernos regionales ${ }^{7}$.

No obstante, y a pesar de las diferencias, cabe señalar que, en ambos casos, se ha producido una especie de regulación intermedia (mesocorporatista) de la política ambiental, en la que se han desarrollado dinámicas de negociación en distintos niveles: en el caso de Francia, entre los responsables de la Administración nacional y los grupos organizados de la sociedad civil; en el caso español, entre la Administración central y la de los gobiernos regionales, y entre todos ellos y la Comisión Europea, que es en última instancia la que ha tenido, y tiene, la última palabra en la aprobación de la lista definitiva de los $\mathrm{LIC}^{8}$.

La comparación realizada sobre el proceso de elaboración de la lista de LIC en Francia y España permite interrogarnos sobre la influencia real del sistema político-administrativo de cada país en la buena gestión de los espacios naturales. De hecho, la mayor extensión de la lista española, gracias al impulso intervencionista de las administraciones regionales, no significa que deba esperarse en España una mejor gestión de la Directiva Hábitat en los espacios de la red Natura 2000, sino más bien lo contrario. $\mathrm{Al}$ ser espacios definidos sin consenso previo con la población local, el programa de conservación se percibe como impuesto desde fuera, con lo que surgen resistencias, por parte de los grupos afectados, a la hora de desarrollar las acciones previstas en la Directiva.

El análisis de esta fase inicial del programa Natura 2000 nos ha llevado a plantear la hipótesis de que en la buena gestión de una política ambiental influye más el contexto socioeconómico local del espacio a proteger, que el sistema administrativo encargado de definirla e implementarla. De ahí que el propósito de los estudios realizados en las zonas seleccionadas, enmarcadas todas ellas en sistemas político-administrativos diferentes y contextos locales bien distintos, haya sido analizar las condiciones sociales que favorecen o desfavorecen la aplicación de las políticas de conservación de la naturaleza en territorios concretos. Dado el carácter exploratorio de esta segunda fase de la inves-

7. Esta impresión es corroborada por la OCDE en su evaluación (realizada en 2004) de la política ambiental española, donde se pone de relieve la insuficiencia de la participación social en la aplicación de las políticas de conservación de la naturaleza en España. El informe muestra con precisión la falta de comunicación entre las autoridades (nacionales y regionales), por una parte, y la sociedad civil, por otra, y recomienda hacer un esfuerzo significativo para desarrollarla (OCDE, 2004).

8. Este proceso de intermediación recuerda otros similares ya analizados en Francia por la sociología del trabajo (Reynaud, 1997; Filatre y De Tersac, 2005) y podría enmarcarse en las prácticas mesocorporatistas estudiadas en diversas políticas sectoriales (Cawson, 1984; Pérez Díaz, 1985; Moyano y Pérez Yruela, 1985; Espina, 1991). 
tigación, nuestra ambición ha sido definir y formular mejor dicha hipótesis, de modo que pueda ser contrastada empíricamente en investigaciones posteriores.

\section{Análisis de los casos de estudio}

En la segunda parte de la investigación, hemos estudiado, en siete áreas integradas en la red Natura 2000, el problema de la implementación de las políticas de conservación de los espacios naturales en España y Francia. Más precisamente, el análisis se ha centrado en el problema que surge cuando se ha de combinar, de un lado, el interés general (abstracto e ideal) de protección de la naturaleza y, de otro, la variedad de intereses particulares (concretos y reales) que caracterizan la gestión de los recursos naturales en el nivel local. La base del problema radica en que los espacios naturales no sólo tienen una dimensión ecológica, sino también una dimensión social y económica, al ser el soporte de usos recreativos (ocio) y de actividades productivas (extracción de áridos, agricultura, alamedas, etc.) y culturales (las riberas de ríos como patrimonio local). Por ello, la conservación de estos espacios naturales es tanto un reto social (desarrollo sostenible), como un problema político (conciliar intereses diversos). La diversidad y complejidad de los problemas de conservación del medio ambiente en esos entornos ponen de relieve la importancia que adquieren las políticas elaboradas a ese respecto.

De acuerdo con ese planteamiento general, los objetivos principales del estudio han sido los siguientes: comprender las dinámicas sociales que surgen en torno a la conservación del medio natural, considerando que las políticas públicas responden a una lógica de beneficios difusos para la sociedad y de costes concretos para sus destinatarios, e identificar los criterios sociales que explican el modo de implementar las políticas ambientales en cada área.

Los resultados que se exponen en este trabajo son los correspondientes a cuatro de los siete estudios realizados en el marco de la tesis doctoral de Sylvia Becerra, ya comentados en el apartado de metodología de este artículo: uno, en la región francesa de Languedoc-Roussillon, en las lagunas de Bages-Sigean; otro, en Cataluña, en el parque natural de Els Aiguamolls de l'Empordà (Girona), y los dos restantes en Andalucía (en las marismas de la bahía de Cádiz y en las marismas del río Palmones en su desembocadura en la bahía de Algeciras, frente al Estrecho de Gibraltar).

\section{Las lagunas de Bages-Sigean (región Languedoc-Roussillon, Francia)}

El primer estudio trata de las lagunas de Bages-Sigean situadas en la región sureña francesa de Languedoc-Roussillon. Es un amplio espacio natural que se extiende por los municipios de Narbonne, Bages, Sigean, Gruissan y Port la Nouvelle. Sus valores naturales han sido reconocidos a nivel europeo a raíz de su integración en la red Natura 2000, con objeto de contrarrestar los problemas de contaminación de las aguas y de la presión demográfica debida al 


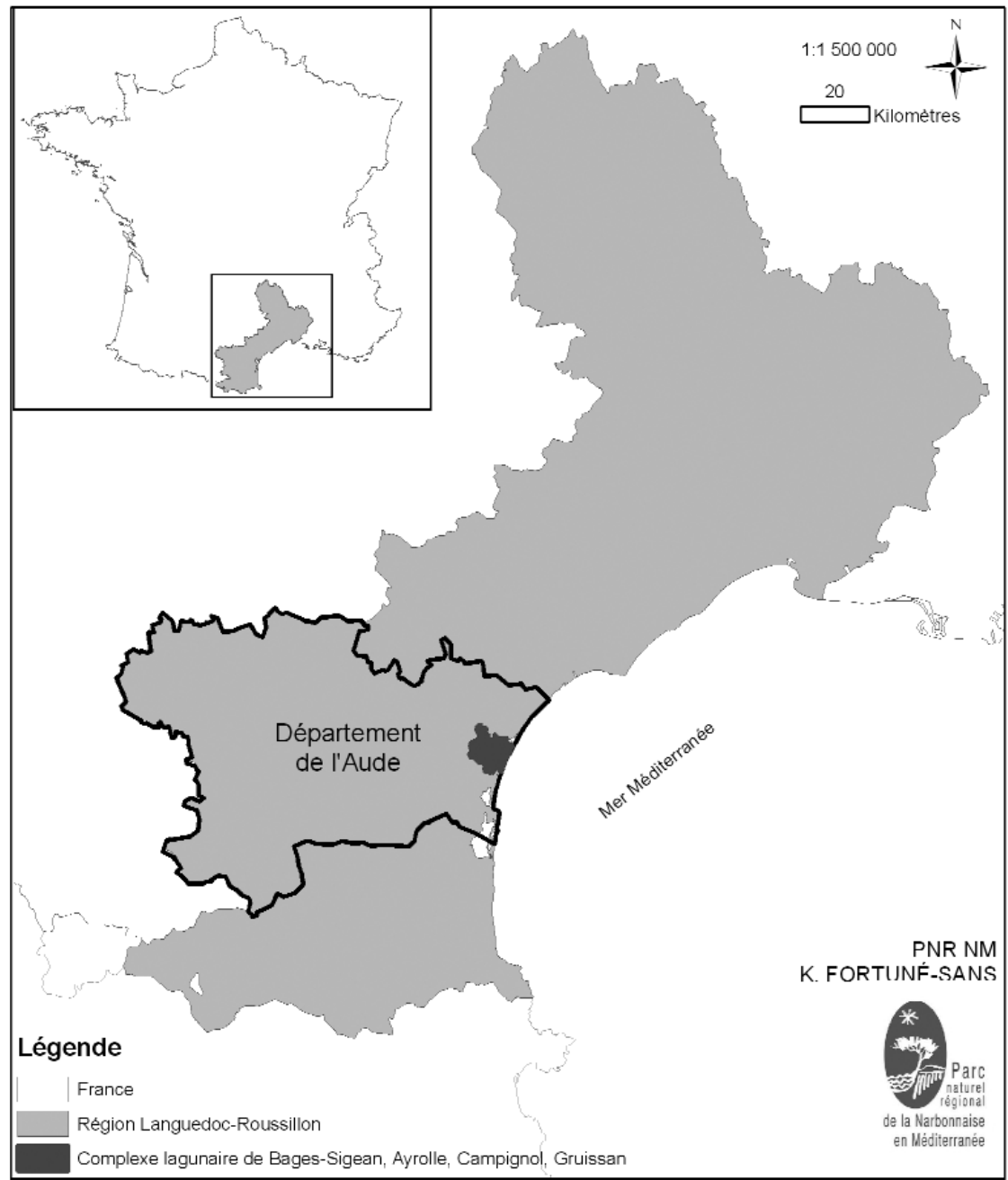

Mapa 1. Localización de las lagunas de Bages-Sigean.

turismo, mayormente en verano (es la tercera región turística francesa) (ver mapa 1).

El Estado ha desempeñado un papel muy importante en el tipo de ordenación del territorio de todo el litoral mediterráneo a partir de los años sesenta, de tal modo que puede decirse, sin ánimo de caer en la exageración, que la política de desarrollo urbanístico conocida como mission racine ha contribuido a destruir gran parte de las zonas húmedas del litoral. Pero, al contrario de lo ocurrido en otras zonas, la zona húmeda de Bages-Sigean ha sido bastante bien 
preservada, como lo prueba el hecho de que sólo existe una estación balnearia con fines turísticos, la de Gruissan. A pesar de esta situación relativamente buena, las asociaciones ecologistas proponen año tras año programas más avanzados de conservación que nunca han llegado a aplicarse y que han sido objeto de frecuentes debates, dado que los ayuntamientos, en particular el de la ciudad de Narbonne, se oponen a realizar una protección demasiado estricta del paraje natural, por temor a que pueda limitar futuros proyectos turísticos, como la creación de una zona residencial. La zona húmeda de Bages-Sigean es hoy el corazón de un parque natural creado en 2003 (JO n 292 de 18 de diciembre del 2003) y denominado Narbonnaise en Méditerrannée (8.000 ha, 36.500 habitantes y 27 municipios). Entre los años 1998 y 2000, este proyecto de parque natural se configuró como alternativa de desarrollo sostenible a las figuras más radicales de conservación propuestas hasta entonces por los grupos ecologistas para salvaguardar el paraje de Bages-Sigean. Con la aprobación del Parque Natural, el paraje obtuvo en 2005 el distintivo "Ramsar», recibiendo así el reconocimiento internacional por sus valores ecológicos.

El éxito aparente del proyecto de parque natural se explica por su contenido y por la evolución que siguió desde sus planteamientos iniciales, habiendo sido un acicate para conciliar los intereses de los diversos actores implicados: las asociaciones conservacionistas, los grupos económicos de la comarca, los políticos locales y los usuarios del litoral. De hecho, aun antes de que se superaran las dificultades administrativas surgidas ante el Ministerio de Medio Ambiente para la aprobación definitiva del proyecto de parque natural, los actores locales dotaron a la zona de una especie de carta de intenciones en la que se fijaban los objetivos que debía perseguir la gestión del espacio natural y las actuaciones necesarias para alcanzarlos, hasta el punto de que, cuando se aprobó el Parque, ya venían aplicándose muchas de las medidas incluidas en sus normas de constitución. El proyecto de parque también sirvió para obtener fondos europeos, con lo que constituyó un importante incentivo para la implicación de los ayuntamientos.

No obstante, la coalición de actores implicados inicialmente en el proyecto experimentó cambios sensibles que hasta pusieron en riesgo la viabilidad de la propia coalición. Al principio, el proyecto ligaba la conservación del espacio natural a un programa de desarrollo local, lo que permitió conciliar los intereses de grupos procedentes de muy diversos sectores, algunos de ellos sin vinculación alguna con la conservación de las zonas húmedas, sino más bien interesados en ellas por razones de carácter económico. Sin embargo, más tarde, tras la contaminación de las lagunas producida por el temporal de 1999, se produjo un cambio importante dentro de la coalición, en la cual predominó la posición de los ecologistas, quienes consiguieron que las actuaciones inicialmente previstas en el proyecto de parque se centraran entonces en la conservación de los humedales de Bages-Sigean. A raíz de ese cambio en los objetivos del proyecto, surgieron unos problemas de tal magnitud dentro de la coalición que, si bien no llegaron a romperla, le hicieron perder el impulso inicial, lo cual retrasó la aprobación del parque natural. Por ejem- 
plo: las asociaciones de cazadores, integradas en la coalición, rechazaron la idea de hacer de las lagunas de Bages-Sigean el eje del futuro parque natural, por considerar que cambiaba las orientaciones iniciales del proyecto y relegaba a un segundo plano la idea de unir la conservación al desarrollo local; asimismo, criticaron el sesgo ecologista que adquiría el proyecto, al haberse propuesto la inclusión de las lagunas en el programa europeo Natura 2000. Las resistencias de estos grupos recibieron el apoyo de algunos grupos políticos, lo que significó sacar a la luz las diferencias que hasta entonces se habían mantenido dentro de la coalición?

Toda la dinámica social y política surgida durante el largo proceso de gestación y aprobación del Parque Natural ha sido, además, una ocasión para innovar y definir normas comunes de actuación, lo cual ha permitido conciliar los intereses generales y locales a la hora de conservar el medio ambiente y ha hecho de la conservación un instrumento compatible con el desarrollo local. La asociación gestora del proyecto de parque desempeñó en este sentido un papel de mediador, coordinando las intervenciones de unos y otros grupos e interpretando las normas generales de modo que pudieran ser compartidas y aceptadas por todos los actores locales. De hecho, esta asociación es considerada hoy un actor de referencia por todos los grupos implicados y por los poderes públicos con competencia en asuntos relativos al medio ambiente.

En resumen, podemos concluir que las estrategias de los actores locales han influido en la conservación de las zonas húmedas de Bages-Sigean en función de varios factores: la correlación de fuerzas entre los distintos grupos sociales; su relación con la Administración central y los intereses dominantes en cada momento, unos intereses que, como se ha observado, pueden cambiar a lo largo del proceso - por ejemplo, con ocasión del temporal que propició el ascenso de las ideas ecologistas dentro de la coalición, al hacer visible la situación de deterioro del espacio natural y plantear la necesidad de recuperar el interés por las actuaciones de conservación, sin menoscabar el potencial de desarrollo de la zona.

\section{Els Aiguamolls del Empordà (Cataluña, España)}

El segundo caso de estudio corresponde a la gestión del parque natural de Els Aiguamolls de l'Empordà (Alto Ampurdán), situado en la bahía de Roses, cerca de la frontera natural de los Pirineos (mapa 2). Este caso es significativo del papel desempeñado por los actores locales a la hora de proteger un paraje natural y, sobre todo, de su capacidad para crear normas de gestión adecuada y

9. Concretamente, los cazadores recibieron el apoyo del partido de oposición al Gobierno (socialista) de uno de los cinco municipios ribereños. La región Midi-Pyrénées está gobernada por una coalición de centro-derecha, mientras que el gobierno provincial (equivalente a nuestra Diputación) es socialista. Corresponde al Gobierno regional ejecutar el desarrollo del proyecto de parque natural en las zonas húmedas de Bages-Sigean. 

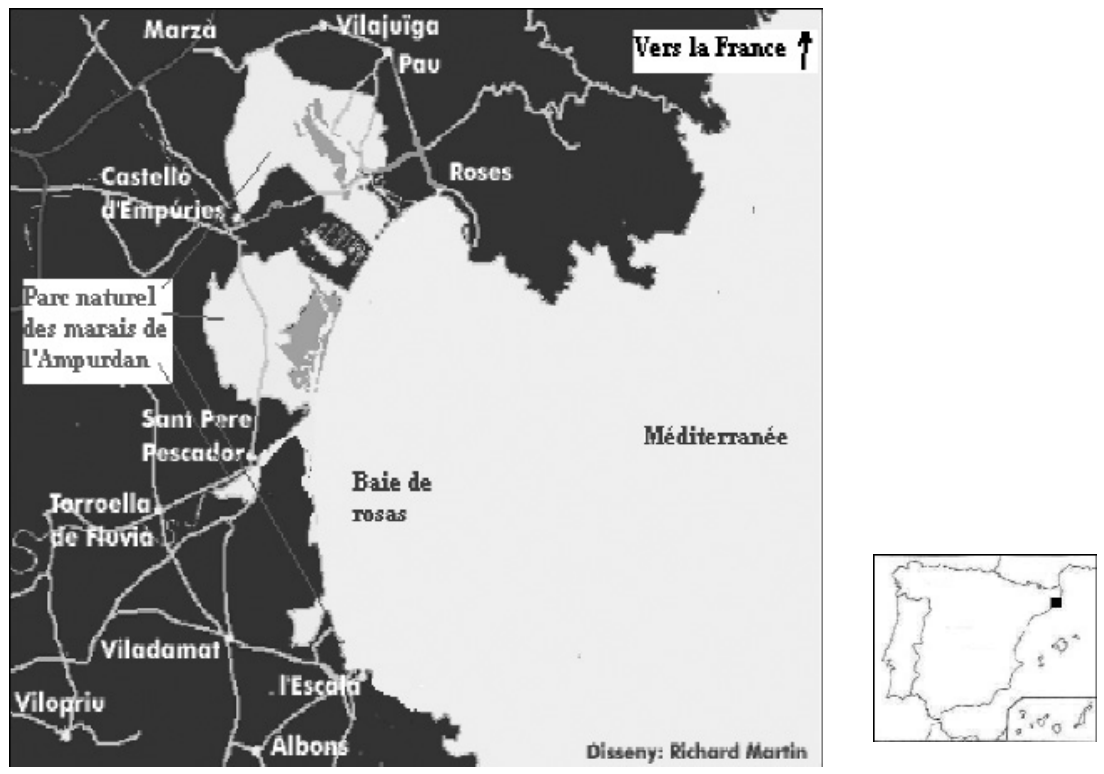

Mapa 2. Localización de las marismas de la bahía de Roses.

compatible con las actividades locales. El parque se creó en 1989, a raíz de una intensa movilización social que duró siete años.

La coalición de actores para la conservación del paraje de Els Aiguamolls de l'Empordà reunió, en 1976, a asociaciones ecologistas, personalidades del mundo científico local e individuos enamorados del paraje natural, así como a habitantes de los municipios cercanos de Figueras y Girona. Pero la deficiente vertebración del movimiento ecologista y la debilidad del tejido asociativo de los usuarios locales (cazadores y agricultores) provocaron un desfase entre, de un lado, los representantes que actuaban en los foros de debate (principalmente científicos y ecologistas) y, de otro, los comportamientos y las prácticas de los distintos grupos sobre el territorio.

Asimismo, y en relación con la creación del parque, numerosos conflictos en torno a la gestión del agua provocaron el enfrentamiento entre los intereses económicos de los agricultores y los intereses generales de la administración del parque, dirigida por el líder local de los ecologistas: en ese momento, aún no existía un interés colectivo que permitiera a todos converger en la necesidad de gestionar el recurso hídrico de un modo consensuado. Para los usuarios tradicionales, la creación del parque significaba una ruptura con la cultura tradicional, así como restricciones no siempre justificadas a su uso y disfrute por la población (sobre todo, desde la perspectiva de los cazadores). Por su parte, los agricultores, mal organizados todavía en sindicatos, no fueron capaces de expre- 
sar sus reivindicaciones de manera colectiva, con lo que perdieron, de hecho, capacidad de actuación y de influencia en el debate ${ }^{10}$.

Para el movimiento ecologista, la creación del parque significó una victoria: la de la ecología sobre los intereses urbanísticos, y ello aportó al movimiento cierto reconocimiento social y político a nivel regional. Además, teniendo en cuenta la limitada participación de la Generalitat de Catalunya en la organización y gestión del parque, su director (un líder ecologista local, como hemos comentado) creó su propio sistema de conservación, apoyándose en los recursos locales y en experiencias de gestión de las zonas húmedas observadas en Francia (en concreto, del parque nacional de Camargue). Ese margen de autonomía de la dirección del parque se tradujo también en dar prioridad a la inundación controlada de las lagunas (para contribuir a su conservación) frente a los intereses agrícolas (contrarios a ello), lo que creó tensiones con los agricultores y provocó el desarrollo de ciertas prácticas al margen de la legislación vigente (por ejemplo, corta de árboles en los canales).

Por su parte, los ayuntamientos de los municipios afectados adoptaron posturas diferentes en función de los costes y los beneficios que representaba el parque natural para el desarrollo local. Para ciertos municipios, como Castelló d'Empúries, el parque era un elemento de diversificación de la oferta turística, al crear un potencial para el desarrollo del turismo rural como alternativa al tradicional turismo de «sol y playa». Para otros municipios más pequeños, cuya economía estaba basada en la agricultura, el parque significaba más limitaciones que posibilidades de desarrollo. A pesar de todo, en los últimos años, el contexto local ha jugado a favor de la participación de los usuarios en la gestión equilibrada del parque natural. Dos elementos parecen haber permitido la pacificación de las relaciones entre los usuarios y la Administración local del parque: el cambio del director conservador y la llegada de los fondos europeos.

El lanzamiento de un programa de ayudas agroambientales, al ser incluido el parque en el convenio Ramsar (sobre zonas húmedas), coincidió efectivamente con la llegada de un nuevo director, considerado por los actores locales como más atento a las reivindicaciones sociales y a los intereses de los usuarios que a las demandas de los ecologistas. Las medidas agroambientales no sólo han compensado la pérdida de rentabilidad de los cultivos o el cambio de las prácticas agrícolas, sino que también han permitido a los agricultores adquirir un nuevo estatus como "cogestores» del parque natural. El dispositivo contractual, implícito en el programa agroambiental, ha permitido, además, transformar las restricciones (consideradas como limitaciones) en oportunidades para integrar nuevas perspectivas de gestión de las explotaciones agrarias y para establecer nuevas relaciones de cooperación

10. Además, la gestión del agua por parte de la Administración local creó desigualdades entre los agricultores, según que las tierras lindaran o no con las reservas integrales del parque o con las zonas voluntariamente inundadas por los grupos ecologistas, lo cual provocó disensiones internas. 
entre agricultores y movimiento ecologista (Garrido-Fernández, 2000 y 2006). De hecho, el programa agroambiental constituye la posibilidad de definir normas de gestión compartidas y aceptadas por todos los grupos implicados, así como la posibilidad de equilibrar la relación de fuerzas existentes a nivel local, una relación que, dominada hasta ese momento por los grupos ecologistas, había obstaculizado la conservación efectiva de las zonas húmedas. Desde entonces, los agricultores han tenido un papel esencial en la protección del espacio natural.

En resumen, la conservación del parque natural de Els Aiguamolls de l'Empordà es hoy el resultado de una acción equilibrada y negociada entre ecologistas y agricultores, cuyos intereses respectivos han convergido tras numerosos conflictos. Este caso expresa la capacidad de los actores locales para crear una coalición de intereses por sí mismos, si bien hay que reconocer que el contexto europeo (encarnado en la forma de las ayudas ligadas al programa agroambiental) ha contribuido de forma significativa a impulsar una dinámica positiva de colaboración, pacificando las tensiones sociales. El caso estudiado expresa también la capacidad de aprendizaje de los actores locales y su reflexividad, es decir, la capacidad para aprovechar sus respectivas experiencias de gestión del medio natural (sean buenas o malas), para, a partir de una reflexión crítica sobre ellas, comprometerse en la búsqueda de un equilibrio entre la conservación del medio ambiente — ligada al discurso actual del desarrollo sostenible- y el mantenimiento de los cultivos, lo que convierte las prácticas agrícolas locales en símbolo de una identidad que no se quiere perder ante la imposición de los programas europeos de medio ambiente (Becerra, 2003b).

\section{Las marismas de la bahía de Cádiz y del río Palmones (Andalucía, España)}

Podemos distinguir dos categorías de espacios protegidos por la política andaluza de conservación de espacios naturales: por una parte, los espacios poco poblados, de gran extensión y muy conocidos por la opinión pública, que reciben ayudas públicas para impulsar el desarrollo local y que, de hecho, generan beneficios económicos ${ }^{11}$; por otra, los espacios protegidos, que no tienen mucha presencia en los medios de comunicación ni generan beneficios económicos para las poblaciones afectadas, estando su conservación afectada por la existencia de una gran presión demográfica sobre ellos.

Los dos estudios realizados en Andalucía pertenecen a la segunda categoría de espacios naturales protegidos. Son espacios poco atendidos por la Administración pública; las reglamentaciones que se aplican en ellos no están bien diseñadas y son de difícil aplicación, y la intensidad de los usos tradicionales que se ejercen en ellos tiene impactos importantes en su conservación.

11. Es el caso, por ejemplo, del parque de Doñana, ampliamente conocido por la opinión pública y beneficiario de un fuerte apoyo financiero, técnico y administrativo por parte de la Administración central y andaluza. 

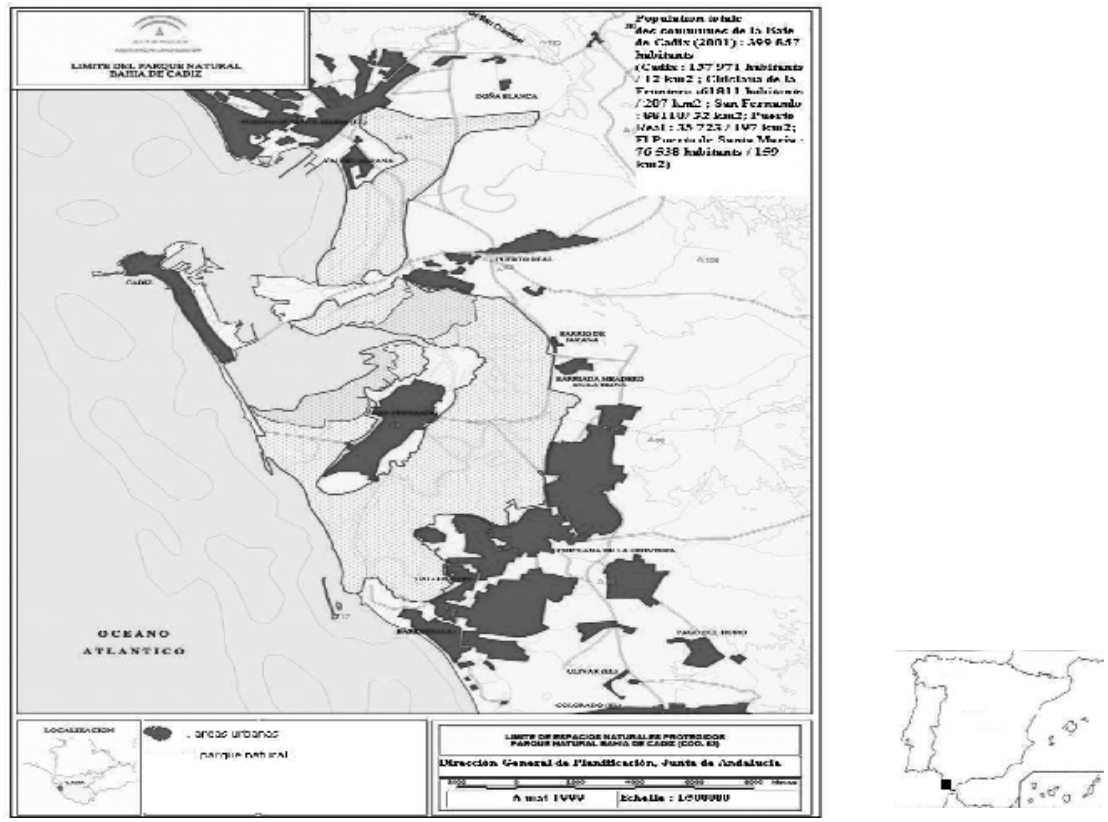

Mapa 3. Localización del parque natural de la bahía de Cádiz (Andalucía).

Creemos que el estudio de estos dos casos confirma la hipótesis de que la conservación de estos espacios naturales con poco potencial económico se explica menos por el dispositivo administrativo, que por las características locales del contexto de actuación.

El primer caso (mapa 3), las marismas gaditanas, es una inmensa zona fangosa periurbana afectada por la aglomeración ciudadana de Cádiz (la tercera de Andalucía), una aglomeración que cuenta con 400.000 habitantes distribuidos en cinco municipios (Cádiz, San Fernando, Chiclana, Puerto de Santa María y Puerto Real).

La configuración geográfica de este espacio natural limita la expansión de los municipios, ya que su conservación ha añadido restricciones administrativas a las perspectivas de crecimiento de ciudades como Cádiz o San Fernando. En tal contexto, la conservación del espacio natural ha pasado a un segundo plano en las preocupaciones, tanto de los municipios, como de la Administración regional, que se ha interesado más por otros asuntos relacionados con los problemas económicos de una zona especialmente afectada por el desempleo y la reconversión industrial ${ }^{12}$.

12. Es una zona históricamente afectada por conflictos relacionados con la industria naval (astilleros y empresas accesorias, sobre todo), que, de forma recurrente, estallan al socaire de crisis o reconversiones (la última de ellas ha sido protagonizada por los trabajadores de la 

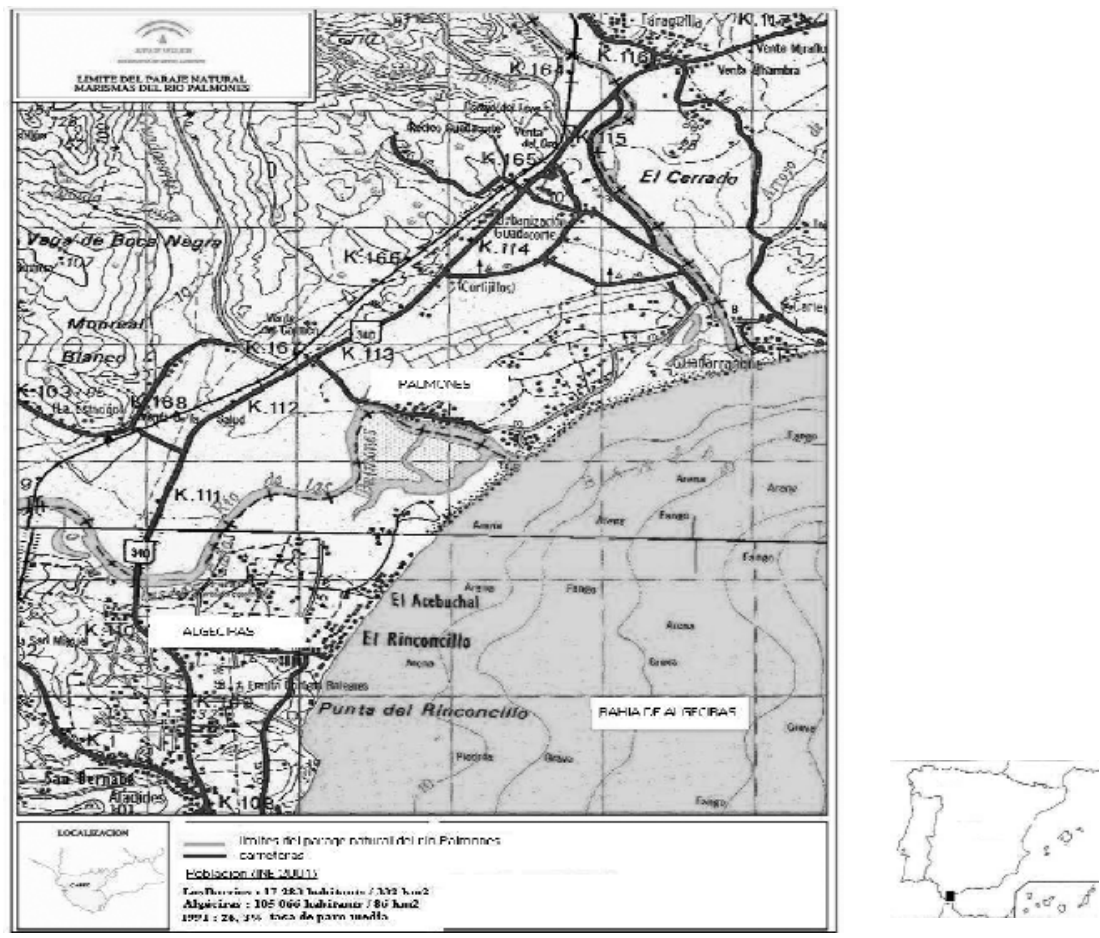

Mapa 4. Localización del paraje natural del río Palmones (Andalucía).

El segundo lugar estudiado se sitúa en la bahía de Algeciras (mapa 4), frente al estrecho de Gibraltar. La zona de marismas del río Palmones constituye una de las últimas escalas para las aves en su migración hacia África, y está sometida a los impactos de una importante zona industrial implantada en los años cincuenta en los márgenes de los ríos que desembocan en la bahía algecirense.

En el caso de los espacios naturales de la bahía de Cádiz, las políticas de conservación se han enfrentado a múltiples problemas, aunque es verdad que han contribuido a evitar el caos en la zona, asegurando el nivel de agua en las marismas y frenando incluso la especulación sobre los terrenos del litoral. Ante la complejidad de las interacciones que suscitan los temas relativos al medio ambiente en esta zona, las normativas ambientales han representa-

multinacional Delphi, instalada en Puerto Real). No sorprende, por tanto, que las preocupaciones de los responsables políticos y de la población en general estén más centradas en estos asuntos que en la conservación del parque natural. 
do aquí un escaso poder de presión sobre los grupos de intereses locales. $\mathrm{Ni}$ siquiera la ampliación del espacio protegido en aplicación de la Directiva europea Hábitat ha tenido efectos en la lógica de gestión local de la zona húmeda, de tal modo que, en este extenso paraje natural, se desarrollan usos, ordenaciones y proyectos no compatibles con su conservación. Nuestro estudio revela que esta situación de anomia (más bien de caos) se debe a un sistema de planificación muy frágil, sometido a enormes presiones y dotado de pocos medios.

Como muestra, algunos botones: el número de mariscadores aficionados, así como el número de barcos en los canales del parque, han aumentado de manera incontrolada; las actividades de pesca y marisqueo, consideradas desde siempre compatibles con el medio natural, se han convertido hoy en un factor de sobreexplotación de los recursos, así como en un elemento de desaparición de algunas especies y destrucción de hábitats; recordemos también que se construyó un centro comercial en medio del parque natural y una planta depuradora; los proyectos de desdoblamiento del ferrocarril o el proyecto de segundo puente de acceso a la ciudad de Cádiz (que no tratamos aquí), también se están realizando a pesar de las normativas existentes de protección de la naturaleza. Estos problemas son ejemplo de las adaptaciones y rodeos que realizan los actores locales a la normativa conservacionista en un espacio natural intensamente humanizado. En efecto, en el contexto local de las marismas gaditanas, la presión social se ejerce en el sentido del crecimiento económico y no en favor de la creación de una coalición de actores favorable a la aplicación de las medidas de conservación vigentes. No obstante, cabe señalar que los grupos ecologistas desempeñan un papel de control ciudadano que, a modo de "pepitos grillos», contribuyen, con sus denuncias, a atenuar los incumplimientos de las distintas administraciones.

Respecto al segundo caso de estudio, el pequeño espacio natural de Palmones, la concentración de fuertes y diversos intereses (económico, militar, político, ecológico, turístico...) y las interdependencias estructurales que han existido desde siempre entre la amplia variedad de usuarios de un territorio tan reducido como éste, también han conducido a una situación de caos. Durante los años setenta, el uso industrial dominante en la zona condicionó los demás usos de las marismas, lo cual produjo una considerable reducción de la zona húmeda original y la modificación de la configuración tradicional de los usos al proponérseles puestos de trabajo fijo en las industrias a los pescadores y mariscadores que vivían de los recursos naturales del estuario. Desde entonces, los usos pesqueros y de marisqueo se han convertido en usos esencialmente recreativos o de complemento de renta, que se ejercen a menudo al margen de la legalidad, debido a que la situación de grave crisis del empleo en la comarca transforma esas actividades en salidas de carácter informal para muchas familias. Mal controladas, dichas actividades de pesca y marisqueo han contribuido a deteriorar los recursos naturales de la zona. Asimismo, la percepción negativa que tiene la población sobre la pequeña zona de marismas (reforzada por los efectos sobre ella de 
la implantación del polígono industrial) explica el poco cuidado que se tiene del paraje y la aparición de usos ilegales o de proyectos poco compatibles con la conservación del mismo.

Además, los municipios muestran posiciones contradictorias. Así, el de Algeciras, por ejemplo, desarrolla, por una parte, su política de medio ambiente (Instituto Municipal de Conservación de la Naturaleza) y, por otra, apoya el proyecto de extensión del puerto sobre las riberas del río Palmones (zona de actividades logísticas del puerto de Algeciras). En ese contexto, la protección efectiva del espacio natural no se debe a la intervención de los poderes públicos, sino a la movilización de los ecologistas y a las normas informales establecidas por los propios usuarios locales, que se organizan para garantizar la explotación de los recursos desde una lógica comercial (complemento de rentas) y una lógica patrimonial (conservación de sus cultivos). Esta situación ha generado una interesante (e interesada) convergencia entre ecologistas y usuarios locales: nuevas alianzas, aún frágiles, se construyen progresivamente, gracias a una especie de «inteligencia ecológica» donde se intenta combinar la conservación de los recursos naturales y el desarrollo de la comunidad local ${ }^{13}$.

Pueden sacarse varias enseñanzas de estos dos casos de estudio en Andalucía, lo cual permite enriquecer el análisis sobre las modalidades de acción colectiva de los actores locales en un espacio natural protegido, pero sometido a una fuerte presión económica y social. En primer lugar, en ambos casos se comprueba que las normativas sobre protección de la naturaleza no son capaces de resistir las dinámicas locales, principalmente en lo que se refiere a los intereses económicos, ya que chocan con la coalición de actores «desarrollistas» formada por los políticos locales, los industriales y los promotores turísticos.

En segundo lugar, esta relación de fuerzas favorables a los intereses económicos se explica por la situación de crisis del empleo a nivel local, pero también por factores político-institucionales. El sistema legislativo español, en el que se superponen normativas diversas en los distintos niveles administrativos, multiplica las dificultades para aplicar en el nivel local la legislación relativa a la protección del medio ambiente (Aguilar-Fernández, 1997). La superposición de normas locales, regionales, nacionales y europeas implica una dispersión de competencias entre los distintos niveles administrativos, y la presencia de una densa red burocrática que hace difícil identificar a los agentes encargados de la aplicación y el seguimiento de cada normativa. En ese escenario, el proceso de aplicación de las normas relacionadas con la conservación de la naturaleza aparece rodeado de incertidumbres en su ejecución, debido a los amplios márgenes de maniobra de que disponen los actores locales (sean públi-

13. Ver, en este sentido, el trabajo realizado por el IESA (2006) (con metodología cualitativa y cuantitativa) sobre actitudes y comportamiento de la población ante los efectos sobre el medio ambiente del complejo industrial instalado en la zona de la bahía de Algeciras. 
cos o privados) para soslayar el rigor de la legislación ambiental en beneficio de sus respectivos intereses (Becerra, 2001) ${ }^{14}$.

En tercer lugar, en esta situación, la dinámica social en los lugares estudiados muestra que la capacidad de influencia y el poder de los actores locales posicionados a favor de la protección del espacio natural son relativamente escasos, debido a los factores citados anteriormente. Es un hecho que la conservación de los recursos naturales no interesa lo suficiente en las dos zonas analizadas como para ser capaz de estructurar formas sólidas de cooperación entre los actores que intervienen en los territorios respectivos. No obstante, y a pesar de ello, surgen formas de acción colectiva en torno a la protección de las zonas húmedas, si bien menos por conciencia ecológica que por garantizar el mantenimiento de unos recursos naturales que son el eje principal de las actividades tradicionales y que constituyen, de hecho, un factor complementario de renta para numerosas familias ${ }^{15}$.

En cuarto lugar, los grupos ecologistas locales desempeñan un papel muy importante en la aplicación de las normativas, así como en el control de los usos, dado que conocen los problemas prácticos que pivotan sobre estos espacios protegidos. Su papel de control se ejerce en forma de denuncias, y también a través de acciones informativas y de concienciación ciudadana, así como de negociaciones con los usuarios para elaborar proyectos conjuntos como el de la gestión sostenible de las salinas en la bahía de Cádiz. Este tipo de estrategia presenta la ventaja de tener en cuenta la diversidad de los intereses en juego y de conocer sobre el terreno los usos tradicionales que, año tras año, han influido en la conformación del paisaje y en la gestión de las marismas.

Por último, el estudio de estos dos casos nos permite sostener que las normativas ambientales permiten impulsar la interacción entre los diferentes actores sociales, más que orientar los comportamientos individuales (o sectoriales). Estas normas funcionan, en realidad, como sistemas de intermediación entre grupos guiados por intereses diferentes, pero que, gracias a ellas (para algunos una amenaza, para otros una oportunidad), se ven obligados a entrar en procesos de debate y negociación y a pensar en cómo hacer compatible el desarrollo y la conservación de la naturaleza.

$\mathrm{Al}$ igual que en los casos anteriores, los resultados empíricos del estudio realizado en estas dos zonas de marismas gaditanas permiten formular con mayor precisión la hipótesis inicial, según la cual la aplicación de las normativas medioambientales está condicionada por la configuración social existente en los espacios naturales. Con el concepto de «configuración» entendemos más concretamente las interacciones que se producen entre los municipios, los inte-

14. Los salineros o los piscicultores de la bahía de Cádiz, por ejemplo, se basan en el Plan de Ordenación del Parque Natural (PORN) para mostrar la legitimidad de sus prácticas, a pesar de que contradicen la legislación vigente sobre la conservación del litoral.

15. Los mariscadores, por ejemplo, se ven obligados a informarse sobre la calidad del agua y sobre los posibles riesgos de contaminación que pueden poner en peligro sus capturas, así como a controlarlas y medir los riesgos que corren. 
reses económicos y el interés general de conservar la naturaleza, creando un escenario complejo en el que las normas ambientales se superponen sobre los métodos tradicionales de gestión a nivel local. En ese contexto local, y en ausencia de un apoyo firme y decidido por parte de las administraciones regional (competente en los espacios protegidos) y nacional (competente en la legislación relativa al litoral), sólo el interés de los usuarios directos de estos espacios naturales (afectados por su degradación) y el activismo de los grupos ecologistas locales, parecen factores lo suficientemente fuertes como para estructurar acciones de tipo colectivo que posibiliten una adecuada aplicación de las normativas ambientales.

\section{Conclusiones}

Los casos analizados sugieren que el elemento determinante de la protección de los espacios naturales debe buscarse en el nivel local, es decir, en la configuración de actores que intervienen en el territorio y en las normas informales que ellos producen. Cabe hablar, por tanto, de una especie de "poder local» en la protección de los espacios naturales, que interacciona de forma cooperadora o conflictiva con las intervenciones públicas. $\mathrm{El}$ "poder local», tal como lo mencionamos aquí, se relaciona menos con el tema del gobierno local, tan estudiado por los sociólogos, y más con las estrategias de organización y coordinación de los actores locales y sus efectos sobre el medio ambiente. Hablar de "poder local» implica, de hecho, reconocer la autonomía de estos actores para movilizarse a favor o en contra de las políticas públicas y de las normativas en las que se concretan en cada territorio.

El análisis de las semejanzas y diferencias entre los diversos casos estudiados, sean franceses o españoles, permite poner de relieve, en primer lugar, la existencia de sistemas autónomos de gestión del medio natural a nivel local: cada uno con su propia organización, sus propias normas, y su propia identidad. La cohesión interna de estos sistemas sociales deriva del modo específico como los actores implicados aprovechan y ponen en valor los recursos naturales de su territorio. La creación de normas locales de gestión capaces de cubrir los huecos dejados por la normativa pública o de adaptarlas a cada contexto, contribuye a la construcción de cierta «autonomía local». En efecto, la organización de los usuarios y otros actores locales que intervienen en el medio natural, se basa en normas específicas que compiten o complementan las normas públicas de carácter más general. Sea voluntaria o no, esta especie de autonomía local no puede interpretarse como simple producto de comportamientos espontáneos de la población como reacción a políticas impulsadas desde fuera. En realidad, la mencionada «autonomía local» traduce, a nuestro parecer, relaciones previas de interdependencia, solidaridad o conflicto entre actores, y expresa también el proceso identitario que los liga al medio natural.

En segundo lugar, podemos añadir que las características socioeconómicas, políticas y ecológicas del espacio natural determinan en cierta medida la aplicación de las políticas de conservación de la naturaleza en cada territorio, al 
influir en la capacidad de los actores locales para movilizarse. El «poder» de estos actores reside, sobre todo, en su aptitud para inventar reglas propias de gestión del espacio natural, así como en su capacidad para formar coaliciones favorables o no a la aplicación de las medidas públicas de conservación. El modo como la población local usa el medio natural, y las experiencias de cooperación entre actores locales, pueden inducir la creación de coaliciones sociales, ciertamente contingentes y frágiles, pero cuyo rasgo característico es su capacidad para movilizarse en pro del mantenimiento o la restauración de un determinado espacio natural.

En tercer lugar, las normas públicas no sólo significan una forma de control sobre la población, sino que también desempeñan un papel de estructuración social en el nivel local, al propiciar que los distintos grupos de intereses confronten sus puntos de vista y se vean impelidos a coordinar sus actividades y actuaciones. En este sentido, las normativas de conservación del medio ambiente tienen un papel simultáneo de activación y regulación de los conflictos, movilizando vínculos sociales entre actores que hasta entonces no habían estado dispuestos a negociar ni a definir un interés común, vínculos que facilitan después el desarrollo de nuevas formas de cooperación entre ellos.

En cuarto lugar, la eficacia de las políticas públicas de conservación de la naturaleza depende, en gran medida, de que los responsables de su ejecución tengan en cuenta el modo como se organizan a nivel local los actores implicados en los asuntos ambientales; más concretamente, de su capacidad para saber interpretar las normas informales que guían el comportamiento de los actores locales y que son expresión de la autonomía e identidad de la población. Esto implica considerar a la población local no como destinataria pasiva de estas políticas, sino como sujeto activo cuya cooperación es necesaria para poder aplicarlas con garantía de éxito. La conservación de la naturaleza depende de que pueda generarse a nivel local coaliciones entre, de un lado, los actores implicados en el uso y la conservación del espacio natural y, de otro, los responsables públicos encargados de aplicar la correspondiente normativa.

En definitiva, pensamos que la eficacia del derecho es limitada, pero no debido a la inercia de las autoridades en el control de su aplicación, sino al hecho de que no se tiene en consideración las "otras» normas, es decir, esas normas informales producidas por la población local y que intervienen de forma decisiva en la gestión de los espacios naturales. La eficacia de las medidas (reglamentarias o incentivadoras) de protección de la naturaleza se basa en la capacidad de crear o desarrollar nuevos vínculos sociales con los actores del espacio natural. Pero ha de basarse también en la identificación e integración de las normas de carácter informal que guían el comportamiento de la población. Ya sean normas que deriven de relaciones conflictivas o de carácter cooperativo entre actores locales, tomarlas en consideración constituye un elemento decisivo en la aplicación de las políticas de conservación de la naturaleza, ya que constituyen instrumentos de gran utilidad para propiciar que la población acepte, o al menos no rechace, las medidas en las que se concretan esas políticas en cada territorio. 


\section{Referencias bibliograficas}

Aguilar-FernándeZ, S. (1997). El reto del medio ambiente: Conflictos e intereses en la politica medioambiental europea. Madrid: Alianza Universidad.

Aguilar-Fernández, S.; Font, N.; Subirats, J. (coords.) (1999). Politica ambiental en España: Subsidiariedad y desarrollo sostenible. Valencia: Tirant lo Blanch.

AlPhANDERY, P.; ForTIER, A. (1999). "Natura 2000: le dispositif français á l'épreuve de la réalité sociales». En: REMY, E. (coord.). La mise en directive de la nature: De la directive Habitats aux prémices du réseau Natura 2000. Rapport au Ministére de l'Environnement, p. 71-113.

BECERRA, S. (2001). «La gestion intégrée des zones humides à l'épreuve de l'autonomie des acteurs locaux». Environnement et Société, vol. 26, p. 35-50.

- (2003a). Protéger la nature: Politiques publiques et régulations locales en Espagne et en France. Tesis de doctorado de sociología. Université de Toulouse-Le Mirail. Département de sociologie de l'UFR Sciences Espaces et Sociétés. CERTOPCNRS, conjuntamente con la Universidad Autónoma de Barcelona, 584 p. Disponible en: http://tel.archives-ouvertes.fr/tel-00133021.

- (2003b). «La protection des zones humides du littoral catalan». En: GENDrON, C.; Vaillancourt, J. G. (dirs.). Développement durable et participation publique. Montréal: FIDES-PUM, p. 325-342.

- (2005). "L'efficacité des politiques de la nature en question. Le cas de l'estuaire du fleuve Palmones (Andalousie, Espagne)». En: Filatre, D.; TERSSAC G. de (coords.). Les dynamiques intermédiaires au cour de l'action publique. Toulouse: Éditions Octares.

Billaud, J. P.; Alphandery, P.; Fortier, A.; Pinton, F. (2002). «La nature en directive: l'exemple du réseau Natura 2000». En: PERRIER-CORNET, Ph. (dir.). Repenser les campagnes. París: Éditions de l'Aube-DATAR, p. 239-258.

Cawson, A. (1985). Organized Interests and the State: Studies in Mesocorporatism. Londres: Sage.

Charles, L.; Kalaora, B. (1999). «Natura 2000, loi chasse, la France et l'Europe. Exception ou allergie française à l'environnement?». Nature Sciences Société, vol. 7, no 1, p. 62-63.

EsPINA, A. (1991). Concertación social, neocorporatismo y democracia. Madrid: Ministerio de Trabajo.

Filatre, D.; Terssac, G. de (coords.) (2005). Les dynamiques intermédiaires au cour de l'action publique. Toulouse: Éditions Octares.

GARRIDO-FERNÁNDEZ, F. (2000). La cuestión ambiental en la agricultura: actores sociales y politica agroambiental en España. Málaga: Unicaja.

- (2006). "Los agricultores, como actores de la política agroambiental. Un enfoque multidimensional». Papers, no 81, p. 37-62.

GARRIDO-FERnÁNDEZ, F.; MOYANO, E. (2004). «Agricultura, sociedad y medio ambiente en la España actual». En: Marrón, Ma J.; GArCiA-FERnÁndEZ, G. (coords.). Agricultura, medio ambiente y sociedad. Madrid: Serie Estudios del Ministerio de Agricultura, Pesca y Alimentación, p. 29-56.

IESA (2005). Actitudes, valores y comportamiento de la población sobre los efectos ambientales del complejo industrial de la bahía de Algeciras. Córdoba: IESA-CSIC (monografía).

IZCARA, S. P. (1999). "El déficit de implementación de las directivas medioambientales de la Unión Europea». Revista Internacional de Sociología, no 24, p. 95-116. 
JiMÉNEZ, M.; MoYANO, E. (2005). Los andaluces y el medio ambiente. Sevilla: Consejería de Medio Ambiente de la Junta de Andalucía.

Jollivet, M. (1997). Vers un rural postindustriel. Rural et environnement dans huit pays européens. París: L'Harmattan.

ManN, P. (1991). L'action collective: Mobilisation et organisation des minorités actives. París: Armand Colin.

MOYANO, E. (2005). «Nuevas orientaciones de las políticas europeas de desarrollo rural. A propósito del reglamento FEADER». Revista de Fomento Social. INSAETEA. Córdoba, vol. 60, no 238, p. 219-242.

MOYANO, E.; PÉREZ YRUELA, M. (1985). «La estructura corporativa de la agricultura española». Papers, no 24, p. 119-143.

NeVERS, J. Y. (2002). «Les politiques de protection des espaces naturels, avantages et coûts sociaux». En: Gendron, C.; VAILlanCourT, J.-G. (dirs.). Développement durable et participation publique. Montréal: FIDES-PUM.

OCDE (2005). Examens environnementaux de l'OCDE: France. París.

- (2004). Examens environnementaux de l'OCDE: Espagne. París.

Olson, M. (1965). The Logic of Collective Action. Cambridge: Cambridge University Press.

Padioleau, J. (1993). L'ordre social. Principes d'analyse sociologique. París: L’Harmattan. PÉrEz DíAZ, V. (1984). "Gobernabilidad y mesogobiernos. Autonomías regionales y mesocorporatismo en España». Papeles de Economía Española, no 21, p. 40-76.

REYNAUD, J. D. (1995). "Conflit et régulation sociale, esquisse d'une théorie de la régulation conjointe». En: REYNAUD, J. D. (ed.). Le conflit, la négociation et la règle. Toulouse: Octares Éditions, p. 49-60.

- (1997). Les règles du jeu. L'action collective et la régulation sociale. 3a ed. París: Armand Colin.

Whitby, M. (coord.) (1996). The European Environment and CAP Reform: Policies and Prospects for Conservation. Londres: CAB International. 